\title{
ECOG or Karnofsky Performance Status to Assess Functionality in Glioblastoma Patients Among Different Observers
}

Claudia Panciroli'*, Anna Estival ${ }^{2}$, Giuseppe Lucente ${ }^{3}$, Jose Maria Velarde ${ }^{2}$, Roser García ${ }^{3}$, Laia Vilà ${ }^{2}$, Sira Domenech $^{4}$, Salvador Villà ${ }^{\text {, }}$ Carmen Balañà ${ }^{2}$

${ }^{1}$ Clinical Investigation Unit, Institute Català d'Oncologia, University Hospital Germans Trias i Pujol, Spain

${ }^{2}$ Medical Oncology Service, Institute Català d'Oncologia, University Hospital Germans Trias i Pujol, Spain

${ }^{3}$ Department of Neuroscience, University Hospital Germans Trias i Pujol, Carretera del Canyet s/n, 08916 Badalona (Barcelona), Spain

${ }^{4}$ Institute Diagnostic per l'Imatge, University Hospital Germans Trias i Pujol, Carretera del Canyet s/n, Spain

${ }^{5}$ Radiation Oncology Service, Institute Català d'Oncologia University Hospital Germans Trias i Pujol, Spain

\begin{abstract}
Purpose: Glioblastoma (GB) patients usually have symptoms that affect their functional status, and medical staff, as well as the patients and their caregivers, might have different perceptions about it. The performance status is important to establish a patient's survival prediction and treatment decisions. This study was aimed to explore whether health care providers, patients, and caregivers have a common perception of patients' functional statuses and to investigate in further studies whether the functional scales used in oncology clinical practice are objective tools to evaluate brain tumour patients.
\end{abstract}

Methods: GB patients treated at our Neuro-Oncology Unit were evaluated once using Eastern Cooperative Oncology Group Performance Status (ECOG) and Karnofsky Performance Status (KPS) by a medical oncologist (MO), an independent investigator (II), the patient and the patient's main caregiver.

Results: Fifty patients were enrolled. Concordance in KPS evaluation among the four observers was low (Fleiss' Kappa $=0.354 ; p<0.001$ ) and moderate in ECOG one (Fleiss' $K=0.424 ; p<0.001$ ). Pairing the observers, the concordance between the MO and II was strong (KPS Cohen's Kappa $=0.731$; $p<0.001$, ECOG Cohen's Kappa $=0.741 ; p=0.001$ ), whereas it was poor between the patient and MO (KPS Cohen's Kappa $=0.250 ; p=0.007$. ECOG Cohen's Kappa=0.241; $p=0.009$ ) and between the caregiver and MO (KPS Cohen's Kappa=0.350; $p<0.001$. ECOG Cohen's Kappa=0.346; $p=0.000$ ).

Conclusion: Concordance among the observers was poor or moderate according to the functional scale used. Particularly, caregivers' perception of the patients' functional status was frequently worse than that of the professionals.

Keywords: Karnofsky performance status; ECOG performance status; Glioblastoma patients; Caregivers

\section{Introduction}

Patients with glioblastoma (GB) usually have symptoms at diagnosis that affect their daily life, and the natural history of the disease is marked by progressive neurological and functional deterioration $[1,2]$. Changes in behaviour, emotions, functional status and neurocognitive functions impact not only their daily life but also that of their families $[3,4]$. The family and, especially, the main caregiver play an important role in this process because they usually must take care of the patient constantly in most cases [5].

Evaluation of patients at oncological visits is usually performed using the functional cancer scales Karnofsky Performance Status (KPS) $[1,6]$ or Eastern Cooperative Oncology Group Performance Status (ECOG), the Barthel Index (BI), functional scale for daily activities $[7,8]$, and the Mini-Mental State Examination (MMSE). The latter scale is a quick and simple cognitive test first described for dementia and then used for gliomas to measure a patient's cognitive impairments $[9,10]$, while KPS and ECOG represent widespread metric scores to determine physical functionality despite the tumour, both having predictive value for every cancer [11]. These scales have also shown their prognostic value in neuro- oncology when performed at the first patient assessment [12], as was described by the Radiation Oncology Group (RTOG) with the Recursive Partitioning Analysis (RPA) scale based on performance status, age, type of surgery and altered cognitive status, subsequently being replaced with the MMSE by the European Organization and Research on Cancer Treatment (EORTC) [13].
Thereafter, EORTC confirmed the value of these prognostic subclasses on the first-line trial that established temozolomide concurrent with radiotherapy and then as adjuvant treatment, as the standard treatment in newly diagnosed GB [14-17].

Several factors, including age, performance status, tumour grade, histology and number of prior progressions, are strong predictors of survival in neuro-oncology $[18,19]$. Of these factors, the performance status score, either assessed by the KPS or ECOG scoring system, is consistently a robust prognostic factor to approach therapeutic decisions [20]. Thus, physical performance plays an integral role in the individualization of treatments and disease in malignant glioma [21]. However, these scoring systems fail to fully characterize physical functioning and lack sufficient sensitivity to accurately discriminate among individuals with good performance status (e.g., KPS $>70$; ECOG

*Corresponding author: Claudia Panciroli, Clinical Investigation Unit, Institute Català d'Oncologia, University Hospital Germans Trias i Pujol, Spain, Tel: +34 93497 8925; Fax: +34 93497 8950; E-mail: cpanciroli@iconcologia.net; claudiapanciroli@gmail.com

Received July 03, 2017; Accepted July 24, 2017; Published July 26, 2017

Citation: Panciroli C, Estival A, Lucente G, Velarde JM, García R, et al (2017) ECOG or Karnofsky Performance Status to Assess Functionality in Glioblastoma Patients Among Different Observers. J Mol Biomark Diagn S2: 032. doi:10.4172/2155-9929.S2-032

Copyright: (c) 2017 Panciroli C, et al. This is an open-access article distributed under the terms of the Creative Commons Attribution License, which permits unrestricted use, distribution, and reproduction in any medium, provided the original author and source are credited 
Citation: Panciroli C, Estival A, Lucente G, Velarde JM, García R, et al. (2017) ECOG or Karnofsky Performance Status to Assess Functionality in Glioblastoma Patients Among Different Observers. J Mol Biomark Diagn S2: 032. doi:10.4172/2155-9929.S2-032

Page 2 of 8

0 or 1) $[6,22]$. As a matter of fact, we believe that the performance status scales are not always fully precise to detect the complete range of symptoms due to a neuro-oncologic disease that impacts on the global functioning of patients and their families [23].

In our daily activity, we frequently observe considerable differences in disease perception among healthcare providers, patients, and caregivers concerning the patient's functional status. These aspects have not yet been examined in depth. This leads to different values recorded for the same patient by different professionals at the same visit, creating confusion when establishing a patient's survival prediction and treatment decision making.

This study was aimed to evaluate patients with the previously described scales to test their reproducibility when administered by two different trained professionals with a parallel evaluation also conducted by the patient and the main caregiver. The aim was to test the concordance of the patient's functional status, as assessed by both KPS and ECOG, among the four observers, to investigate in further studies if the scales used in oncology practice are an effective tool to provide an objective evaluation of functionality in brain tumour patients [22], when establishing their survival prediction and oncology treatment.

\section{Methods}

\section{Population}

This prospective study was performed in patients who visited the Neuro-Oncology Unit (UNO) of Catalan Institute of Oncology (ICO)-Badalona. Patients were asked to participate in our study, and we informed them that the results would not interfere with medical treatments and clinical visits. Information about how to apply the KPS and ECOG assessments was also given to participants. Subjects were eligible for the study if they had a diagnosis of GB at any stage of the disease, were accompanied by the main caregiver and could perform a self-assessment of KPS/ECOG. The main caregiver was defined as the person taking care of the patient's needs most of the time. The investigators were the medical oncologist (MO) taking care of the patient's cancer treatment and a $\mathrm{PhD}$ student trained in neurocognitive impairments, as an independent investigator (II).

\section{Study design}

Patients were evaluated once with KPS/ECOG during the same oncological visit. MMSE, BI and a neurological examination were also performed. Both MMSE and BI were administered to every patient by the II, while KPS/ECOG was performed by the MO, II, patient and caregiver. We used the MMSE to evaluate neurocognitive impairments, the BI to identify patient's grade of functional independency, and KPS/ECOG to assess the functional status (Table 1). The participants were asked to provide information about their socio-demographic background through an interview. We classified jobs as "qualified" if the work required higher skills or training previously acquired (i.e., journalist or engineer) and "non-qualified" if the work did not require a skill to be performed (i.e., maid or workman). We classified the educational level as high, middle or low if the participants attended university, secondary school or primary school, respectively. We registered the disease's characteristics, tumour location, oncological treatment scheme and concomitant medications such as antiepileptics (AEDs), anxiolytics, antidepressants, opioids, and corticosteroids. All variables were recorded. To perform our analysis of the concordance between two observers, we compared the MO's evaluation with that of the patient and caregiver because the MO is the professional who makes the final decisions about the patient's care and because of the quite high concordance between the MO and II.

\begin{tabular}{|c|c|c|}
\hline $\begin{array}{l}\text { Karnofsky } \\
\text { Performance } \\
\text { Status (KPS) }\end{array}$ & $\begin{array}{l}\text { Eastern Cooperative } \\
\text { Oncology Group } \\
\text { Performance Status } \\
\text { (ECOG) }\end{array}$ & Description \\
\hline 100 & 0 & $\begin{array}{c}\text { Fully active, able to carry on all pre- } \\
\text { disease performance without restrictions. }\end{array}$ \\
\hline $80-90$ & 1 & $\begin{array}{c}\text { Restricted in physically strenuous activity } \\
\text { but ambulatory and able to carry out work } \\
\text { of a light or sedentary nature, eg., office } \\
\text { work, house work. }\end{array}$ \\
\hline $60-70$ & 2 & $\begin{array}{l}\text { Ambulatory and capable of all self-care but } \\
\text { unable to carry out the previous job. Up } \\
\text { and about more than } 50 \% \text { of waking hours. }\end{array}$ \\
\hline $40-50$ & 3 & $\begin{array}{l}\text { Ambulatory and capable of all self-care but } \\
\text { unable to carry out the previous job. Up } \\
\text { and about more than } 50 \% \text { of waking hours. }\end{array}$ \\
\hline $20-30$ & 4 & $\begin{array}{l}\text { Completely disabled. Cannot carry on } \\
\text { self-care. Totally confined to bed or chair. }\end{array}$ \\
\hline
\end{tabular}

Table 1: Description of Karnofsky Performance Status (KPS) and Eastern Cooperative Oncology Group Performance Status (ECOG) scale measures.

\begin{tabular}{|c|c|c|}
\hline \multirow{2}{*}{ Variables } & Patients & Caregivers \\
\hline & $n=50$ & $\mathrm{n}=50$ \\
\hline \multicolumn{3}{|c|}{ Sex } \\
\hline Male & $25(50 \%)$ & $23(46 \%)$ \\
\hline Female & $25(50 \%)$ & $27(54 \%)$ \\
\hline \multicolumn{3}{|l|}{ Age } \\
\hline$<50$ & $12(24 \%)$ & $16(32 \%)$ \\
\hline$\geq 50$ & $38(76 \%)$ & $34(68 \%)$ \\
\hline \multicolumn{3}{|c|}{ Handedness } \\
\hline Right & $48(96 \%)$ & $50(100 \%)$ \\
\hline Left & $2(4 \%)$ & $0(0 \%)$ \\
\hline \multicolumn{3}{|c|}{ Educational level } \\
\hline None & $3(6 \%)$ & $2(4 \%)$ \\
\hline Low & $30(60 \%)$ & $34(68 \%)$ \\
\hline Middle & $9(18 \%)$ & $5(10 \%)$ \\
\hline High & $7(14 \%)$ & $9(18 \%)$ \\
\hline Not reported & $1(2 \%)$ & $0(0 \%)$ \\
\hline \multicolumn{3}{|c|}{ Job } \\
\hline Non-qualified & $41(82 \%)$ & $35(70 \%)$ \\
\hline Qualified & $8(16 \%)$ & $15(30 \%)$ \\
\hline Not reported & $1(2 \%)$ & $0(0 \%)$ \\
\hline \multicolumn{3}{|c|}{ Tumor characteristics } \\
\hline \multicolumn{3}{|c|}{ Location } \\
\hline Right & $25(50 \%)$ & NA \\
\hline Left & $24(48 \%)$ & \\
\hline Bilateral & $1(2 \%)$ & \\
\hline \multicolumn{3}{|c|}{ Lobe } \\
\hline Frontal & $28(56 \%)$ & \multirow{5}{*}{ NA } \\
\hline Others & $22(44 \%)$ & \\
\hline One & $38(76 \%)$ & \\
\hline More & $10(20 \%)$ & \\
\hline Central & $2(4 \%)$ & \\
\hline \multicolumn{3}{|c|}{ Treatment } \\
\hline Active & $40(80 \%)$ & \multirow[t]{2}{*}{ NA } \\
\hline Non-active & $10(20 \%)$ & \\
\hline \multicolumn{3}{|c|}{ Support } \\
\hline Yes & $47(94 \%)$ & \multirow[t]{2}{*}{ NA } \\
\hline No & $3(6 \%)$ & \\
\hline \multicolumn{3}{|c|}{ Medications } \\
\hline AEDs & $33(66 \%)$ & $0(0 \%)$ \\
\hline Dexamethasone & $20(40 \%)$ & $0(0 \%)$ \\
\hline$<4 \mathrm{mg}$ & $9(45 \%)$ & -- \\
\hline
\end{tabular}




\begin{tabular}{|c|c|c|}
\hline$\geq 4 \mathrm{mg}$ to $<8 \mathrm{mg}$ & $5(25 \%)$ & -- \\
\hline$\geq 8 \mathrm{mg}$ & $6(30 \%)$ & -- \\
\hline Antidepressants drugs & $9(18 \%)$ & $5(10 \%)$ \\
\hline Anxiolytic drugs & $10(20 \%)$ & $8(16 \%)$ \\
\hline Psycho-stimulants & $1(2 \%)$ & $0(0 \%)$ \\
\hline Opioids & $2(4 \%)$ & $0(0 \%)$ \\
\hline
\end{tabular}

Note: Abbreviation: AEDs: Anti-Epileptic Drugs; NA: Not Applicable.

Table 2: Patients and care givers characteristics.

\begin{tabular}{|c|c|c|c|c|}
\hline $\mathbf{n = 5 0}$ & Min & Max & Median & IQR \\
\hline BI & 15 & 100 & 95 & 25 \\
\hline MMSE & 4 & 30 & 25 & 7 \\
\hline Patients' KPS & 40 & 100 & 70 & 30 \\
\hline Caregivers' KPS & 30 & 100 & 70 & 40 \\
\hline MO's KPS & 40 & 100 & 80 & 30 \\
\hline Il's KPS & 40 & 90 & 70 & 30 \\
\hline Patients' ECOG & 0 & 3 & 2 & 1 \\
\hline Caregivers' ECOG & 0 & 3 & 2 & 2 \\
\hline MO's ECOG & 0 & 3 & 1 & 1 \\
\hline Il's ECOG & 1 & 3 & 2 & 1 \\
\hline
\end{tabular}

Note: Abbreviations: BI: Barthel Index; MMSE: Mini Mental State Examination; KPS: Karnofsky Performance Status; ECOG: Eastern Cooperative Oncology Group Performance Status; MO: Medical Oncologist; II: Independent Investigator; IQR: Interquartile Range.

Table 3: Scales' summary descriptive statistics.

\begin{tabular}{|c|c|c|}
\hline $\mathbf{n = 5 0}$ & $\mathbf{K}$ & $\mathbf{p}$ \\
\hline KPS & 0.354 & $<0.001$ \\
\hline ECOG & 0.424 & $<0.001$ \\
\hline
\end{tabular}

Note: Abbreviations: KPS: Karnofsky Performance Status; ECOG: Eastern Cooperative Oncology Group Performance Status; K=Fleiss' Kappa; $p=p$-value.

a) Among the observers.

\begin{tabular}{|c|c|c|}
\hline $\mathbf{n = 5 0}$ & $\mathbf{K}$ & $\mathbf{p}$ \\
\hline MO vs. II KPS scores & 0.731 & $<0.001$ \\
\hline Patient vs. MO KPS scores & 0.25 & 0.007 \\
\hline Caregiver vs. MO KPS scores & 0.35 & $<0.001$ \\
\hline MO vs. II ECOG scores & 0.741 & 0 \\
\hline Patient vs. MO ECOG scores & 0.241 & 0.009 \\
\hline Caregiver vs. MO ECOG scores & 0.346 & 0 \\
\hline
\end{tabular}

Note: Abbreviations: KPS: Karnofsky Performance Status; ECOG: Eastern Cooperative Oncology Group; MO: Medical Oncologist; II: Independent Investigator; $\mathrm{K}=$ Cohen' Kappa; $\mathrm{p}=\mathrm{p}$ value.

b) Pairing the observers.

\begin{tabular}{|c|c|c|c|c|c|c|c|c|c|}
\hline & KPS & 100 & 90 & 80 & 70 & 60 & 50 & 40 & 30 \\
\hline \multirow[t]{5}{*}{$\mathrm{n}=50$} & ECOG & 0 & \multicolumn{2}{|c|}{1} & \multicolumn{2}{|c|}{2} & \multicolumn{2}{|c|}{3} & 4 \\
\hline & $\begin{array}{c}\text { MO's } \\
\text { scores }\end{array}$ & 1 & 15 & 12 & 4 & 10 & 5 & 3 & 0 \\
\hline & Il's scores & 0 & 13 & 11 & 7 & 10 & 5 & 4 & 0 \\
\hline & $\begin{array}{l}\text { Patient's } \\
\text { score }\end{array}$ & 4 & 10 & 10 & 6 & 11 & 8 & 1 & 0 \\
\hline & $\begin{array}{l}\text { Caregivers' } \\
\text { score }\end{array}$ & 3 & 10 & 8 & 8 & 8 & 8 & 3 & 2 \\
\hline
\end{tabular}

c) Distribution of KPS and ECOG scores

Table 4: Concordance of KPS and ECOG scores a) among the observers, and b) pairing the observers. c) shows the distribution of KPS and ECOG scores.

\section{Statistical Analysis}

Statistical analysis was performed using the "Statistical Package for Social Science" (SPSS), version 21.0 and the "R Statistical Programming Language" (R), version 3.1.1. The concordance on the patients' functional status among the four observers was analyzed with
Fleiss' Kappa, using R; while pairing the observers, it was tested with Cohen's Kappa, using SPSS. Cohen's Kappa assesses the concordance only between two raters, whereas Fleiss' weighted Kappa is a robust measure to assess the reliability of agreement between a fixed number of observers, even more than two [24]. That's why in our analysis we used both systems. We considered an almost perfect concordance if Kappa $(\mathrm{K}) \geq 0.81$, a substantial/strong concordance if $\mathrm{K}=(0.80-0.61)$, a moderate concordance if $\mathrm{K}=(0.60-0.41)$, a fair/poor concordance if $\mathrm{K}=(0.40-0.21)$, a slight agreement if $\mathrm{K}=(0.20-0.01)$ and no concordance if $\mathrm{K} \leq 0.00$ [25]. We also reported the MO's and caregiver's KPS score distribution estimated using chi-squared test. A $p$ value $<0.05$ was considered significant.

\section{Results \\ Patient characteristics}

Fifty patients with GB and a caregiver for each one was enrolled in the study. The demographic and clinical characteristics are shown in Table 2. Seventy-six percent of patients were aged $\geq 50$ years, $50 \%$ were males, and $50 \%$ were females. The age cut-off was determined according to RPA. Almost all patients were right-handed (96\%), and most chose to receive family care (94\%). Eighty-two percent of patients had a non-qualified job, and $60 \%$ had a low level of education. The predominant location of the tumour lesion was the frontal lobe $(56 \%)$, and the GB was limited usually to only one lobe (76\%).

Concerning GB involving more than one lobe (20\%), $69 \%$ of patients had a frontal lobe lesion. There were no important differences in terms of the dominant hemisphere involvement (right, 50\%; left, 48\%), and a bilateral affection was present in one patient $(2 \%)$. Ongoing cancer treatments $(80 \%)$ were the first line of treatment in $46 \%$ of patients (temozolomide concomitant with radiation or as adjuvant treatment), the second line in $10 \%$ and the third line in $24 \%$ (bevacizumab, irinotecan or fotemustine). The remaining patients (20\%) received the control (without treatment). Concomitant medications were AEDs in $66 \%$ of patients, corticosteroids in $40 \%$, antidepressants in $18 \%$, anxiolytics in $20 \%$, psycho-stimulants in $2 \%$, and opioids in $4 \%$.

\section{Statistical results of performance status' concordance}

The different summary descriptive statistics are reported in Table $3(n=50)$. We analysed the four observers' report measures using KPS (Tables 4a-4c) (Figures 1a and 1b), we found a fair concordance (Fleiss' $\mathrm{K}=0.354 ; \mathrm{p}<0.001$ ), while moderate when analyzing ECOG scores (Fleiss' $\mathrm{K}=0.424 ; \mathrm{p}<0.001$ ). Furthermore, the concordance between health care providers (MO and II) was strong (KPS Cohen's Kappa $=0.731 ; \mathrm{p}<0.001$. ECOG Cohen's Kappa $=0.741 ; \mathrm{p}=0.001$ ), whereas it was poor between the patient and the MO (KPS Cohen's Kappa $=0.250 ; \mathrm{p}=0.007$. ECOG Cohen's Kappa $=0.241 ; \mathrm{p}=0.009$ ) and between the caregiver and the MO (KPS Cohen's Kappa $=0.350$; $\mathrm{p}<0.001$. ECOG Cohen's Kappa $=0.346 ; \mathrm{p}=0.000$ ). Especially, the caregiver's perception of patients' functionality was mostly worse than the one perceived by the MO $(\mathrm{p}=0.000)$. Figures $2 \mathrm{a}$ and $2 \mathrm{~b}$ shows that in most cases, the MO scores are higher than the caregivers' scores.

\section{Discussion}

We tested the assumption of a discrepancy on the perception of patients' functional status among health care professionals, patients and their families in a cohort of GB patients in our daily clinical context. The variability in KPS/ECOG evaluations among the observers is an important issue, as they have a prognostic value in GB patients, and they could also determine the possibility to enroll subjects in clinical 

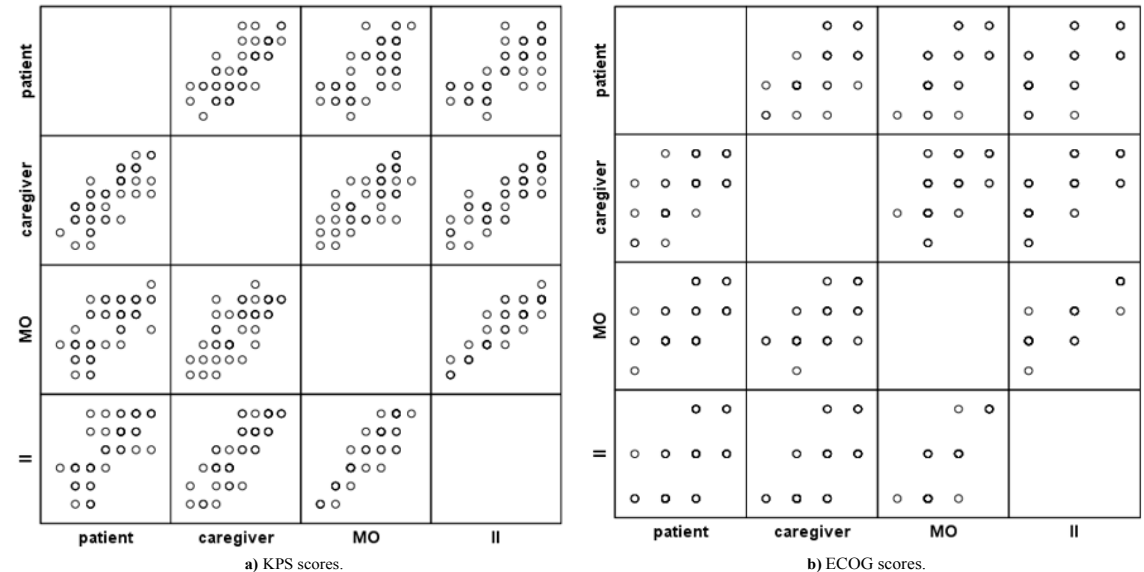

Figure 1: Concordance of a) Karnofsky Performance Status (KPS) scores and b) Eastern Cooperative Oncology Group Performance Status (ECOG) scores among the observers.
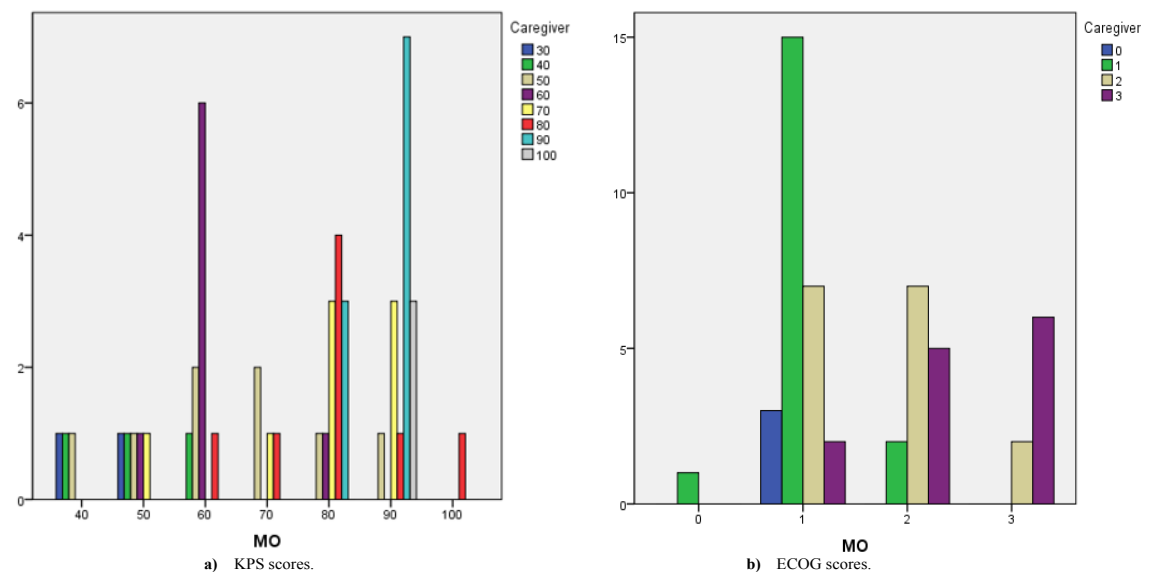

Figure 2: MO's and caregivers' scores regarding their perception of patient's functionality status, assessed by a) Karnofsky Performance Status (KPS) and b) Eastern Cooperative Oncology Group Performance Status (ECOG).

trials, as most of them require an ECOG $\leq 1$ or ECOG $\leq 2$ (KPS from 100 to 80 or 70) [1]. Further, the performance status is important to establish patients' survival prediction and treatment decisions.

Our study found a different concordance of KPS/ECOG scores depending on who performs the scales' evaluation. KPS' concordance among the four observers was poor. The agreement between the II and $\mathrm{MO}$ was strong but fair between $\mathrm{MO}$ and the patients. To support the latter finding, we suspect that patients' cognitive impairments or behavioural changes might cause a distorted functional self-perception $[23,26]$. Additionally, we found that caregivers had frequently a worse patient functional status perception than professionals, and we considered whether the family's overload or burnout aroused by the impaired patient and his/her health condition deterioration could cause the tendency to evaluate worse the patient's functionality $[27,28]$. The caregiver assessment can serve indeed as adequate proxies for patient reports [4]. Thus, we believe the importance that both assumptions might warrant further investigation.

Both ECOG and KPS assess the patients' ability to perform a job [1]. There is a percentage of cases in which patients do not show any physical impairment that affects their functionality $(\mathrm{BI}=100 \%)$; however, due to their low neurocognitive level, they are unable to carry out their previous job. Thus, their ECOG should be evaluated as
2 and the KPS as 70 rather than 0 and 100, respectively. Specifically, we acknowledge that KPS and ECOG do not consider the cognitive and neurological impairments that can affect, for example, the capacity to perform a qualified job but not a non-qualified job. As a matter of fact, neuro-cognitive impairments are important to consider in a job that requires attention and mental efforts. For this reason, we do believe that the fair concordance among the observers should also be explained by the inadequacy to fully evaluate brain tumour patients with KPS/ ECOG, and we highlight that a functional scale adjusted for brain tumour patients is needed.

The main limitation of our study is the limited sample number, which is why concomitant medications, age, educational level, or the lesions' location were not tested as confounding factors. Additionally, we did not record some variables such as neurological impairments, pain severity, stress, burnout, anxiety and depression levels, and no control groups were included. Finally, exploring the agreement among all health care providers involved in patient care, such as radiation oncologists or neurosurgeons, would have also been important to be assessed.

Accordingly, our outcomes suggest that the agreement on the patients' functional status perception among the four observers is fair but higher when using ECOG because the latter scale is shorter 
Citation: Panciroli C, Estival A, Lucente G, Velarde JM, García R, et al. (2017) ECOG or Karnofsky Performance Status to Assess Functionality in Glioblastoma Patients Among Different Observers. J Mol Biomark Diagn S2: 032. doi:10.4172/2155-9929.S2-032

and easier to assess. Therefore, ECOG seems to encompass a wider spectrum of patients' symptoms and to have a better coverage of the caregivers' perception. The cause may be that, in ECOG, there are fewer options, making it easier to score the same.

Medical professionals should carefully screen for the capacity to make treatment decisions concerning brain patients' performance status [1]. Although the KPS was criticized concerning its psychometric characteristics, such as its reliability and insensitivity to neurocognitive impairments, it is still the standard functionality tool used in neuro-oncology practice [29]. However, we remark that none of the performance status' scales are perfect to fully evaluate the patient's functionality, as shown by the different concordance values found among patients/caregivers and professionals.

\section{Conclusion}

Notwithstanding, conclusion should be taken with caution. We did not perform a detailed study but a very preliminary attempt to explore not only professionals' but also caregivers' and patients' point of view regarding patients' functional status. With this aim, we provided additional information for both clinical practice and the planning of future studies. This is important to consider because the functional scales assume different values depending on who administers them.

Compliance with ethical standards All procedures performed in studies involving human participants were in accordance with the ethical standards of the institutional and/or national research committee and with the 1964 Helsinki declaration and its later amendments or comparable ethical standards. Informed consent was obtained from all individual participants included in the study.

\section{References}

1. Martin RC, Gerstenecker A, Nabors LB, Marson DC, Triebel KL (2015) Impairment of medical decisional capacity in relation to Karnofsky Performance Status in adults with malignant brain tumor. Neurooncol pract 2: 13-19.

2. Henriksson R, Asklund T, Poulsen HS (2011) Impact of therapy on quality of life, neurocognitive function and their correlates in glioblastoma multiforme: $A$ review. J Neurooncol 104: 639-646.

3. Minaya FP, Berbis J, Chinot O, Auquier P (2014) Assessing the quality of life among caregivers of patients with gliomas. Neurooncol pract 1: 191-197.

4. Jacobs DI, Kumthekar P, Stell BV, Grimm SA, Rademaker AW, et al. (2014) Concordance of patient and caregiver reports in evaluating quality of life in patients with malignant gliomas and an assessment of caregiver burden. Neurooncol pract 1: 47-54.

5. Mezue WC, Draper P, Watson R, Mathew BG (2011) Caring for patients with brain tumor: The patient and care giver perspectives. Niger J Clin Practice 14: 368-372.

6. Johnson DR, Sawyer AM, Meyers CA, O'Neill BP, Wefel JS (2012) Early measures of cognitive function predict survival in patients with newly diagnosed glioblastoma. Neuro Oncol 14: 808-816.

7. Thomas R, Guerrero D, Hines F, Ashley S, Brada M (1995) Modifying the Barthel Performance Index Score for use in patients with brain tumours. Eur $J$ Cancer Care (Engl) 4: 63-68.

8. Brazil L, Thomas R, Laing R, Hines F, Guerrero D, et al. (1997) Verbally administered Barthel Index as functional assessment in brain tumour patients. J Neurooncology 34: 187-192

9. Arevalo-Rodriguez I, Smailagic N, Roque IFM, Ciapponi A, Sanchez-Perez E, et al. (2015) Mini-Mental State Examination (MMSE) for the detection of Alzheimer's disease and other dementias in people with mild cognitive impairment $(\mathrm{MCl})$. Cochrane Database Syst Rev 3: CD010783.

10. Tombaugh TN, McIntyre NJ (1992) The mini-mental state examination: a comprehensive review. J Am Geriatr Soc 40: 922-935.

11. Chambless LB, Kistka HM, Parker SL, Hassam-Malani L, McGirt MJ, et al.
(2015) The relative value of postoperative versus preoperative Karnofsky Performance Scale scores as a predictor of survival after surgical resection of glioblastoma multiforme. J Neurooncol 121: 359-364.

12. Weller M, Van Den Bent M, Hopkins K, Tonn JC, Stupp R, et al. (2014) EANO guideline for the diagnosis and treatment of anaplastic gliomas and glioblastoma. Lancet Oncol 15: e395-e403.

13. Curran WJ Jr., Scott CB, Horton J, Nelson JS, Weinstein AS, et al. (1993) Recursive partitioning analysis of prognostic factors in three Radiation Therapy Oncology Group malignant glioma trials. J Natl Cancer Inst 85: 704-710.

14. Corn BW, Wang M, Fox S, Michalski J, Purdy J, et al. (2009) Health related quality of life and cognitive status in patients with glioblastoma multiforme receiving escalating doses of conformal three-dimensional radiation on RTOG 98-3. J Neurooncol 95: 247-257.

15. Stupp R, Mason WP, Van Den Bent MJ, Weller M, Fisher B, et al. (2005) Radiotherapy plus concomitant and adjuvant temozolomide for glioblastoma. New Engl J Med 352: 987-996.

16. Stupp R, Hegi ME, Mason WP, Van Den Bent MJ, Taphoorn MJ, et al. (2009) Effects of radiotherapy with concomitant and adjuvant temozolomide versus radiotherapy alone on survival in glioblastoma in a randomised phase III study: 5-year analysis of the EORTC-NCIC trial. Lancet Oncol 10: 459-466.

17. Mirimanoff RO, Gorlia T, Mason W, Van Den Bent MJ, Kortmann RD, et al (2006) Radiotherapy and temozolomide for newly diagnosed glioblastoma: Recursive partitioning analysis of the EORTC 26981/22981-NCIC CE3 phase III randomized trial. J Clin Oncol 24: 2563-2569.

18. Carson KA, Grossman SA, Fisher JD, Shaw EG (2007) Prognostic factors for survival in adult patients with recurrent glioma enrolled onto the new approaches to brain tumor therapy CNS consortium phase I and II clinical trials. J Clin Oncolology 25: 2601-2606.

19. Mauer M, Stupp R, Taphoorn MJ, Coens C, Osoba D, et al. (2007) The prognostic value of health-related quality-of-life data in predicting survival in glioblastoma cancer patients: Results from an international randomised phase III EORTC Brain Tumour and Radiation Oncology Groups, and NCIC Clinical Trials Group study. Br J Cancer 97: 302-307.

20. Oken MM, Creech RH, Tormey DC, Horton J, Davis TE, et al. (1982) Toxicity and response criteria of the Eastern Cooperative Oncology Group. Am J Clin Oncolology 5: 649-655

21. Ruden E, Reardon DA, Coan AD, Herndon JE $2^{\text {nd }}$, Hornsby WE, et al. (2011) Exercise behavior, functional capacity, and survival in adults with malignant recurrent glioma. J Clin Oncol 29: 2918-2923.

22. Jones LW, Cohen RR, Mabe SK, West MJ, Desjardins A, et al. (2009) Assessment of physical functioning in recurrent glioma: Preliminary comparison of performance status to functional capacity testing. J Neurooncol 94: 79-85.

23. Pace A, Villani V, Di Pasquale A, Benincasa D, Guariglia L, et al. (2014) Home care for brain tumor patients. Neurooncol pract 1: 8-12.

24. Landis JR, Koch GG (1977) The measurement of observer agreement for categorical data. Biometrics 33: 159-174.

25. Fleiss JL (1971) Measuring nominal scale agreement among many raters Psychological Bulletin 76: 378-382.

26. Pompili A, Telera S, Villani V, Pace A (2014) Home palliative care and end of life issues in glioblastoma multiforme: Results and comments from a homogeneous cohort of patients. Neurosurg Focus 37: E5.

27. Janda M, Steginga S, Langbecker D, Dunn J, Walker D (2007) Quality of life among patients with a brain tumor and their carers. J Psychosom Res 63: 617-623.

28. Munoz C, Juarez G, Munoz ML, Portnow J, Fineman I, et al. (2008) The quality of life of patients with malignant gliomas and their caregivers. Soc Work Health Care 47: 455-478.

29. Hutchinson TA, Boyd NF, Feinstein AR, Gonda A, Hollomby D, et al. (1979) Scientific problems in clinical scales, as demonstrated in the Karnofsky index of performance status. J Chronic Dis 32: 661-666.

This article was originally published in a special issue, Cancer Biomarkers handled by Editor(s). Dr. Sudhir Srivastava, Cancer Biomarkers Research Group, National Institute of Health, USA; Dr. Shou-Jiang Gao, The University of Texas Health Science Centre at San Antonio, USA; Dr. Kenneth Maiese, University of Medicine \& Dentistry of New Jersey, New Jersey Medical School, USA 